\title{
Decomposition Based on Decision Diagrams
}

\author{
David Bergman and Andre A. Cire
}

Version Post-print/accepted manuscript

Citation Bergman D., Cire A.A. (2016) Decomposition Based on Decision (published version) Diagrams. In: Quimper CG. (eds) Integration of AI and OR Techniques in Constraint Programming. CPAIOR 2016. Lecture Notes in Computer Science, vol 9676. Springer, Cham.

Publisher's Statement The final publication is available at link.springer.com via http://dx.doi.org/10.1007/978-3-319-33954-2_4.

\section{How to cite TSpace items}

Always cite the published version, so the author(s) will receive recognition through services that track citation counts, e.g. Scopus. If you need to cite the page number of the author manuscript from TSpace because you cannot access the published version, then cite the TSpace version in addition to the published version using the permanent URI (handle) found on the record page.

This article was made openly accessible by $U$ of 'T Faculty. Please tell us how this access benefits you. Your story matters. 


\title{
Decomposition Based on Decision Diagrams
}

\author{
David Bergman ${ }^{1}$ and Andre A. Cire ${ }^{2}$ \\ 1 Department of Operations and Information Management, University of Connecticut \\ david.bergman@business.uconn.edu \\ 2 Department of Management, University of Toronto Scarborough \\ acire@utsc.utoronto.ca
}

\begin{abstract}
In recent years, decision diagrams (DDs) have proven useful for solving a variety of optimization problems, often closing long-standing instances from classical benchmarks. This success is primarily driven by a DDs ability to capture structure. This paper exploits this characteristic and proposes a novel solution method which decomposes a problem into highly-structured portions, where the solution set of each portion can be compactly represented using a DD. This technique is applied to a special case of the independent set problem and to unconstrained binary quadratic programming. Preliminary computational results suggest that the proposed decomposition approach can improve upon both standard integer programming models and a single DD approach.
\end{abstract}

Keywords: Decision Diagrams, Decomposition, Network flows.

Pre-print - CPAIOR 2016. The final publication is available at Springer via https://doi.org/10.1007/978-3-319-33954-2_4

\section{Introduction}

A decision diagram (DD) is a graphical data structure originally introduced to compactly represent Boolean functions [1], with several applications in circuit design and formal verification $[14,18]$. In recent years, DDs have also been applied to encode the solution set of discrete optimization problems, serving for a variety purposes such as cut generation in mixed-integer linear programming [5], to enhance propagation in constraint programming [3], and in novel general branch-and-bound procedures for combinatorial optimization problems [8].

In the context of optimization, the successful applications of DDs are ample. Examples include solving long-standing open benchmark instances of the maximum cut problem [8] and of variants of the traveling salesman problem [15]. Moreover, DDs have also been incorporated into state-of-the-art integer programming and constraint programming technology to substantially improve optimality gaps [9-11] and solution times [16] on a number of applications.

One of the key reasons for the successful application of DDs lies in the fact that they are particularly well-suited to capture complete inference for certain problem structures. For example, the size of a DD encoding the feasible solutions of a set covering problem can be bounded by a function of the bandwidth of the 
constraint matrix $[11,17]$. As a result, DD-based methods perform well when the bandwidth of the matrix is small, but tend to lose effectiveness for larger bandwidths. Analogously, DDs provide strong optimization bounds for the maximum clique problem when the underlying graph is dense [9], and can improve solution times in scheduling problem by orders of magnitude depending on the structure of the precedence graph [15].

In this paper we exploit this inherent characteristic of DDs and propose the notion of decision diagram decomposition. The idea is to decompose an optimization problem into distinct subproblems, each capturing some complicating problem structure for which the associated DD is provably small in size. Once equipped with a valid decomposition, the original discrete problem then reduces to finding a path in each DD that mutually agree on the assignment of the decision variables, which can be solved in many ways.

In particular, we propose a methodology in which each DD is assigned a network flow relaxation, thereby transforming the representation from a discrete structure into a mixed-integer linear programming (MILP) model. The network flow relaxations are combined through linking constraints, stated either generally or as problem specific constraints, so as to harness the power of MILP solvers.

The contributions of this work are hence threefold. First, we provide a decomposition approach which captures problem structure in a novel systematic way. Distinct from existing methods, the decomposition of the instance can be based not only on the constraints, but also on the objective function, or on both. Second, the resulting MILP from our methodology effectively yields new extended formulations [4] in a generic way, i.e. MILP models in a higher dimension that can be stronger than other existing models. Finally, our methodology can also be used to improve the robustness of current DD-based methods in constraint programming and operations research, since one can use different DDs to represent different substructures as opposed to a single DD for all the problem.

The paper is organized as follows. Section 2 introduces BDDs, and Section 3 explicitly expresses the decomposition framework. Next, Section 4 provides the MILP formulation for linking the distinct BDDs, and preliminary study cases are presented in Section 5 on the maximum independent set problem and the binary quadratic programming problem. A conclusion is provided in Section 6 .

\section{Decision Diagrams for Binary Optimization}

For the purposes of this paper, we focus on solving binary optimization problems (BOPs), which are of the form $\max \left\{f(x): x \in S, x \in \mathbb{B}^{n}\right\}$, where $n$ is the number of variables, $f$ is any function mapping binary vectors into the set of real numbers, and $S$ is an arbitrarily defined constraint set.

A BDD $B=(U, A)$ is a directed acyclic graph with nodes $U$ and $\operatorname{arcs} A$. The nodes are partitioned into $n_{B}$ layers $L_{1}, \ldots, L_{n_{B}}$, and each node $u \in U$ is in layer $\ell(u) \in\left\{1,2, \ldots, n_{B}\right\}$; thus, $L_{i}=\{u \mid \ell(u)=i\}$. In particular, $L_{1}=\{\mathbf{r}\}$ and $L_{n_{B}}=\{\mathbf{t}\}$, where $\mathbf{r}$ and $\mathbf{t}$ are referred to as the root node and terminal node, respectively. Each arc $a \in A$ has an arc-weight $w(a) \in \mathbb{R}$ and an arc-domain 
$d(u) \in\{0,1\}$, where $a$ is a 0 -arc when $d(a)=0$ and an 1-arc otherwise. An arc $a=(h(a), t(a))$ has head $h(a)$ and tail $t(a)$, with $\ell(t(a))-\ell(h(a))=1$. Each node $u \in U \backslash\{t\}$ is the head of at most one 0 -arc and at most one 1-arc.

We use BDDs is to represent solutions (or partial solutions) to BOPs as $r-t$ paths, where the length of the path corresponds to objective function values through the total weight of the $\mathbf{r}-\mathbf{t}$ path. Let $\mathcal{P}(B)$ be the set of arc-specified $r-t$ paths in $B$. Each layer, with the exception of the terminal layer, is associated with a variable through the injection $\sigma^{B}:\left\{1, \ldots, n_{B}-1\right\} \rightarrow\{1, \ldots, n\}$. For a path $p=\left(a_{1}, \ldots, a_{n_{B}-1}\right) \in \mathcal{P}(B)$, the arc-domains along $p$ yield a partial solution $x(p)=\left(x_{\sigma^{B}(1)}, x_{\sigma^{B}(2)}, \ldots, x_{\sigma^{B}\left(n_{B}-1\right)}\right)=\left(d\left(a_{1}\right), d\left(a_{2}\right), d\left(a_{n_{B}-1}\right)\right)$. Let $\mathcal{X}(p)=$ $\left\{x \in \mathbb{B}^{n}: x_{\sigma^{B}(i)}=x_{i}(p), i=1, \ldots, n_{B}-1\right\}$ be the set of possible completions of partial solutions, and define the solutions of $B$ as $\operatorname{Sol}(B)=\bigcup_{p \in \mathcal{P}(B)} \mathcal{X}(p)$.

Let the weight $w(p)$ of path $p$ be $\sum_{i=1}^{n_{B}^{\prime}-1} q\left(a_{i}\right)$. An exact BDD $B$ for a BOP is one in which $(1) \operatorname{Sol}(B)$ coincides with the set of feasible solutions to the BOP and (2) for each path $p$ and each solution $x \in \mathcal{X}(p), f(x)=w(p)$. Such a BDD encodes all feasible solutions along with their objective function values, so that a longest path, which can be computed in linear time in $|U|$, corresponds to an optimal solution and its length corresponds to the optimal solution value.

To illustrate, consider the BOP $\mathcal{P}^{\prime}$ defined by $\max f(x)=\sum_{i=1}^{5} x_{i}$ subject to $x_{1}+x_{2} \leq 1, x_{1}+x_{3} \leq 1, x_{2}+x_{3} \leq 1, x_{2}+x_{4} \leq 1, x_{3}+x_{4} \leq 1$, and $x_{4}+x_{5} \leq 1$. The optimal solution value is 2 . Figure 1 (a) depicts an exact BDD for this BOP. The variables corresponding to each layer appear on the left of the BDD and dashed/solid arcs correspond to setting variables on that layer to $0 / 1$.

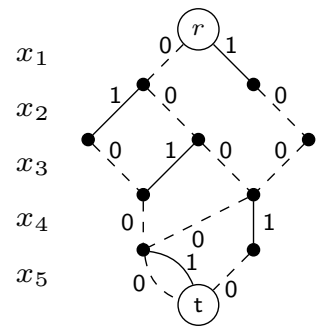

(a)

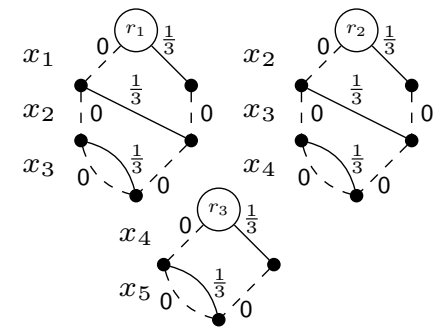

(b)

Fig. 1. (a) Exact BDD and (b) Exact DBDD for example BOP.

\section{Decomposition based on Binary Decision Diagrams}

A decomposition based on binary decision diagrams (DBDD) for a BOP is a collection of BDDs $B_{1}, \ldots, B_{m}$ with the following two properties: (1) $\bigcap_{k=1}^{m} \operatorname{Sol}\left(B_{k}\right)$ coincides with the feasible set of the BOP, and (2) for any feasible solution $x$, the set of paths $p_{k}$ in BDD $B_{k}$ for which $x \in \mathcal{X}\left(p_{k}\right), k=1, \ldots, m$, satisfy 
$f(x)=w\left(p_{1}\right)+\cdots+w\left(p_{k}\right)$. These two conditions enforce that every feasible solution to the BOP corresponds to some path in each of the BDDs, that every vector in $\mathbb{B}^{n}$ which is infeasible does not correspond to any path in at least one of the BDDs, and that the sum of the weights of these paths coincide with the objective function value of that solution. In particular, the latter condition can be enforced by setting the weights of the 1-arc in layer $k$ of each BDD as $f\left(x_{k}\right) / m\left(x_{k}\right)$, where $m\left(x_{k}\right)$ is the number of BBDs which include variable $x_{k}$. Finally, note that a single BDD is a DBDD.

Figure 1(b) depicts a DBDD for $P^{\prime}$ : Any solution is a collection of 3 paths, one in each $\mathrm{BDD}$, for which the variable assignments coincide. The optimal solution $(1,0,0,1,0)$ corresponds to the solid-dashed-dashed/dashed-dashed-solid/dashedsolid path through the BDD with roots $r_{1}, r_{2}, r_{3}$, respectively.

\section{Solving a DBDD}

Given a BDD for a BOP, finding the optimal solution reduces to a longest path computation which, since a BDD is a directed-acyclic graph, can be identified in $\mathcal{O}(|U|)$. The same is not true for a DBDD - even determining whether or not $\bigcap_{k=1}^{m} \operatorname{Sol}\left(B_{k}\right)$ is empty is NP-hard. To establish this, consider the set packing problem, which asks for a binary vector $x$ that maximizes a linear function and that satisfies $A x \leq 1$, where $A$ is a $0 / 1$ matrix. The exact BDD for each constraint has width of 2 [10], the collection of exact BDD for each individual constraint is a DBDD, and finding a common solution would solve the set packing problem.

In order to solve the underlying DBDD optimization problem, we formulate an MILP model based on interpreting the longest path of each BDD as a network flow. Let $B=(U, A)$ be a BDD and consider the MILP model netflow $(B)$ :

$$
\begin{gathered}
\operatorname{netflow}(B)=\max _{\substack{x \in\{0,1\}^{n} \\
y \in[0,1]^{|A|}}}\left\{\sum_{a \in A} w(a) y_{a}: \sum_{a \in A: h(a)=\mathbf{r}} y_{a}=1, \sum_{a \in A: t(a)=\mathbf{t}} y_{a}=1,\right. \\
\left.\sum_{a \in A: t(a)=u} y_{a}-\sum_{a: h(a)=u} y_{a}=0 \forall u \in U, x_{\sigma^{B}(i)}=\sum_{a \in A: \ell(a)=i, d(a)=1} y(a) \forall i\right\}
\end{gathered}
$$

Each arc $a \in A$ has a variable $y_{a}$ and the constraints are the typical network flow constraints which enforce that each feasible solution must correspond to a $\mathbf{r}-\mathbf{t}$ path, with an additional set of constraints that relate the flow variables with the $\mathrm{BOP}$ variables. These constraints enforce that the BOP variable $x_{\sigma^{B}(i)}=1$ if and only if the arc of the path on layer $i$ is a 1-arc. Note that a similar formulation can be created for other forms of BDDs, including zero-compressed BDDs [9], which will be used in the computational results that follow.

It is well known that netflow $(B)$ is an integral polytope so that relaxing the integrality constraints results in a linear programming (LP) for which the corner points are integral. Moreover, if the BDD is exact, the projection onto the $x$-variables is a convex-hull relaxation of the feasible set of the BOP [5]. 
Hence, a DBDD $\left\{B_{k}\right\}_{k=1}^{m}$ can be formulated as an MILP model that combine the polytopes netflow $\left(B_{k}\right)$, which are naturally linked through the variables $x$. In particular, the feasible solutions to netflow $\left(B_{k}\right)$ is $\operatorname{Sol}\left(B_{k}\right)$, and therefore the conjunction of the polytopes (represented by the combined model) yields an extended formulation for the BOP. One of the key advantages is that each $B_{k}$ may capture some combinatorial structure that is non-trivial to encode as linear constraints, thus yielding better MILP models. In order to make the approach scalable, each BDD in the DBDD should be as small as possible.

\section{Study Cases}

We examine two problem classes as preliminary study cases which exemplify how problems can be decomposed into structured subproblems with limited-size BDDs. All experiments ran on an Intel(R) Core(TM) i7-4770 CPU @ 3.40GHz processor, 32 GB RAM, using IBM ILOG CPLEX 12.6 (one thread).

Independent Sets on Social Networks. The application that motivated the design of DBDDs was finding independent sets in social network graphs. Let $G=$ $(V, E)$ be an undirected graph with vertices $V=\{1, \ldots, n\}$ and edge set $E \subseteq V \times$ $V$. An independent set $I$ is a subset of $V$ such that no two vertices in $I$ are adjancent to each other, i.e. $i, j \in I$ if $(i, j) \notin E$. The maximum independent set problem asks for an independent set $I$ with the largest cardinality. This problem is typically written as the BOP $\max \left\{\sum_{i \in V} x_{i}: x_{i}+x_{j} \leq 1 \quad \forall(i, j) \in E, x \in \mathbb{B}^{n}\right\}$.

In graphs representing social networks, independent sets play a key role in identifying interpersonal relations [6], in game theoretical models for the provision of goods [13], and as a measure of fairness [20] and diversity [19]. Here we focus on a particular type of social network graph denoted by relaxed caveman graphs. A caveman graph represents large groups of mutually adjacent connections with sporadic links to other groups, as depicted in Figure 2. Independent sets in such graphs are used, e.g., in procedures for detection of communities [2]. The decomposition in this case consists of building the exact BDD for each

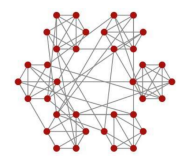

Fig. 2. Relaxed caveman graph. Picture from Judd et al [20].

denser component (the "caves"), and then using single edge inequalities to enforce that the endpoints of the redirected edges cannot both be in an independent set. The BDD for a single clique has width $2[17,9]$ and, upon removal of one edge, the width of each layer grows by at most a factor of 2 . Therefore, if $k$ edges are removed, the size of the exact BDD is at most $2^{k}$, although in general will 
be much smaller. Since there are typically few interconnections in this class of graphs, the BDDs will be of practical size.

For our experiments, we constructed random graphs specified by a triple $(c, s, p)$ : First, $c$ cliques of size $s$ are generated, and then the endpoints of the edges in a clique are randomly assigned to a vertex in another clique with probability $p$. We considered $c=10, s \in\{15,20, \ldots, 40\}$, and $p \in\{0.10,0.15, \ldots, 0.50\}$. Three instances per triple $(c, s, p)$ were generated, yielding in total 175 instances. We compared three approaches: a clique-based MILP formulation for the problem [9], the MILP generated from the DBDD, and Cliquer [21], a specialized method for the problem. The clique-based MILP method exploited the clique structure imposed by the caves. A time limit of 30 minutes was set in all cases.

Figure 3(a) depicts the number of solved instances over time, and shows that the DBDD MILP is more robust then all other methods combined. Figure 3(b) compares times between the DBDD and Cliquer, where the size of a point indicates the number of vertices in each cave $(s)$ and the color gradation indicates the density. In particular, DBDD performs better for any instance that can be solved in more than 10 seconds by both methods, and it is particularly more effective for larger problems with a higher density between caves.

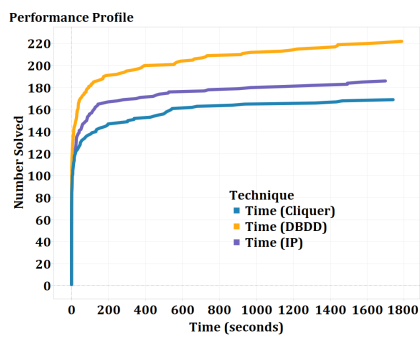

(a) Performance profile

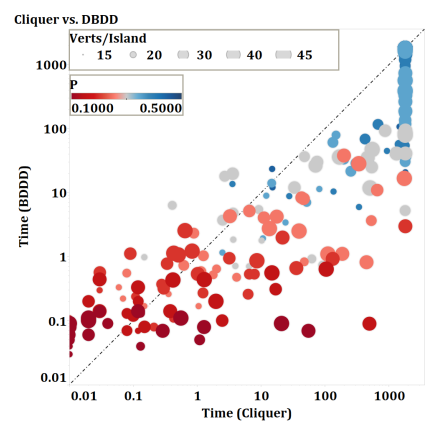

(b) Cliquer vs. DBDD

Fig. 3. Results on relaxed caveman graphs.

Unconstrained Binary Quadratic Programming (UBQP). We present a case where the objective function is decomposed as opposed to the constraint set. A instance of UBQP is specified by an objective function matrix $Q \in \mathcal{S}^{n}$, where $\mathcal{S}^{n}$ is the set of symmetric $n \times n$ matrices (i.e., $\forall i, j \in\{1, \ldots, n\}, q_{i, j}=q_{j, i}$ ). The UBQP is defined as $\max \left\{x^{T} Q x: x \in \mathbb{B}^{n}\right\}$. For notation purposes, let $x \mid k$ be the partial solution of $x \in \mathbb{B}^{n}$ on only the first $k$ indices: $x \mid k=\left(x_{1}, \ldots, x_{k}\right)$.

An exact BDD $B$ for BOP can be compiled with a top-down approach based on a dynamic programming (DP) formulation for the problem, as presented in Bergman et al $[7,8]$. DP models in our context are defined by a state $s($.$) and$ a value function $v($.$) , which will be encoded as nodes of the BDD and as length$ of arcs, respectively. For the UBQP with $Q=\left\{q_{i, j}\right\}$, the state is defined as 
$s\left(x^{\prime} \mid k+1\right)_{j}=0$ if $j \leq k+1$, and $s\left(x^{\prime} \mid k\right)_{j}+2 \cdot q_{k+1, j} \cdot x_{k+1}^{\prime}$, if $j>k+1$. The value function is written as $v\left(x^{\prime} \mid k+1\right)=v\left(x^{\prime} \mid k\right)+x_{k+1}^{\prime} \cdot s\left(x^{\prime} \mid k\right)_{k+1}$. Intuitively, the value in the $j$ th coordinate of the state represents the marginal effect of setting $x_{j}=1$ given the values assigned to $x_{1}, \ldots, x_{k}$. We omit the proof for brevity, but note that they are similar to the DP model of the maximum cut problem [8]. Figure 4 depicts an exact BDD for an objective function matrix $Q^{\prime}$.

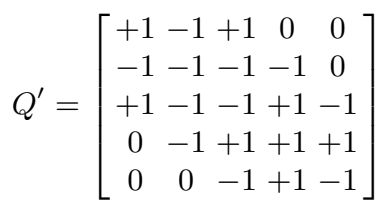

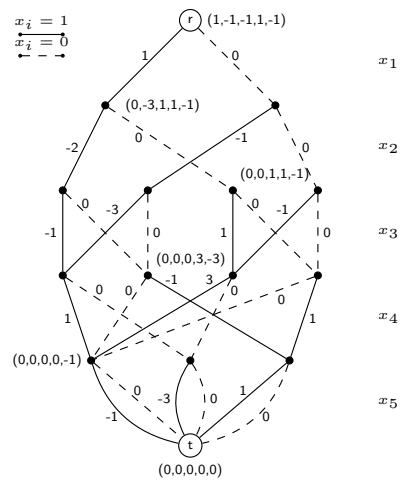

Fig. 4. Objective function matrix for an UBQP instance with an exact BDD. States are indicated on nodes in which the path corresponding to solution $(1,0,1,1,0)$ traverses.

Given a graph $G=(V, E)$, a path decomposition of $G$ is a sequence of subsets $V_{i} \subseteq V$ for which (1) $\forall e \in E, \exists V_{i}$ s.t. $e \subseteq V_{i}$ and (2) $\forall i \leq j \leq k, V_{i} \cap V_{k} \subseteq V_{j}$. The width of a path decomposition is one less that the maximum cardinality set, $\max _{i}\left|X_{i}\right|-1$. The path-width, $p w(G)$, of a graph $G$ is the minimum width over all path decomposition. For a symmetric $n \times n$ matrix $Q$, let $G(Q)=\left(V, E^{\prime}\right)$ be the graph with $E^{\prime}=\left\{(i, j) \in E: q_{i, j} \neq 0\right\}$. We state the following result, which follows a similar proof as in previous works bounding the size of DDs [17].

Theorem 1. Let $Q$ be an $n \times n$ symmetric matrix. There exists an exact $B D D$ for the $U B Q P$ defined on $Q$ for which $w(B) \leq 2^{p w(G(Q))-1}$.

Consider $Q^{\prime}$ in Figure 4. A path decomposition for $G\left(Q^{\prime}\right)$ consists of vertex sets $\{1,2,3\},\{2,3,4\},\{3,4,5\}$, proving that there exists a BDD with width bounded by $2^{3-1}=4$. The BDD in Figure 4 is such a witness.

Theorem 1 provides a bound on the size of the width of BDDs based on the pathwidth of $G(Q)$. This indicates that for matrices whose corresponding graphs have a limited pathwidth, a DD-based approach will work well. However, if $Q$ does not possess such a characteristic, the DD can grow exponentially large. We exploit this idea to decompose the matrix $Q$ as follows: fix $p, 1 \leq p \leq n$ as the desired pathwidth for each element of the decomposition, and let $m=\lceil n / p\rceil$ be the number of elements created. Define $Q^{k}$ by, for $i \leq j, q_{i, j}^{k}=q_{i, j}$ if $k(p-1) \leq$ $i<k p$ and $j \geq i$, and $q_{i, j}^{k}=0$ otherwise, with the elements in indices with 
$i>j$ defined so that $q^{k}$ is symmetric (i.e., $q_{i, j}^{k}=q_{j, i}^{k}$ ). Each $Q^{k}$ satisfies that $p w\left(G\left(Q^{k}\right)\right) \leq p$, thereby limiting the size of the exact BDD for each $Q^{k}$.

In order to test the effectiveness of the approach, random symmetric matrices were generated, with $n=40$ and fixed $Q$ to have bandwidth $L \in\{10,20,30,40\}$, with 10 instances per configuration. Each $q_{i j}$ took a value uniformly at random from $\{-5,-4, \ldots, 4,5\}$ if $i \neq j$, and the diagonal entries were set as $q_{i, i}=$ $-1 \cdot \sum_{j \neq i} q_{i, j}$ if $|i-j| \leq L$, and 0 otherwise.

Our goal is to show how DBDDs can improve upon integer programming methodology. Figure 5(a) presents a performance profile, depicting the effect of varying $p$, the pathwidth of each BDD in the decomposition. Each line corresponds to a $p$ and the resulting number of BDDs. Figure 5(b) presents a plot depicting the average time to solve instances with $L \in\{10,12,14,16\}$. We provide a comparison of the IP linearization implemented in CPLEX [12], namely $\min \left\{\sum_{i, j} q_{i, j} y_{i, j}: x_{i}+x_{j}-1 \leq y_{i, j} ; x_{i}, x_{j} \geq y_{i, j} ; x_{i}, y_{i, j} \in\{0,1\}\right.$, all $\left.i, j\right\}$, with a single exact BDD and the DBDD with $p=4$. A time limit was set to 600s.

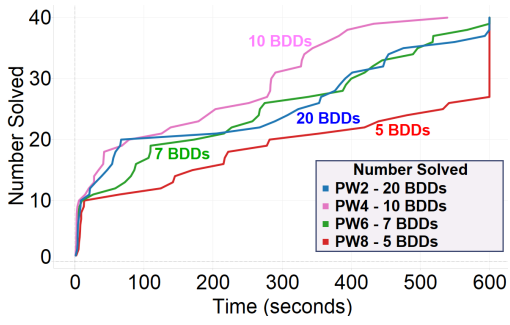

(a) Performance profile (vary $p$ )

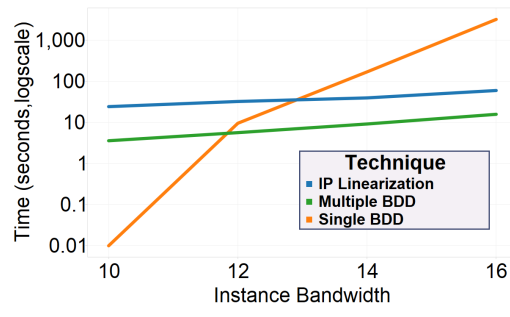

(b) (Average) Time to solve

Fig. 5. UBQP Results.

Figure 5 shows how a single exact BDD approach is impractical because of the exponential growth in its size as $L$ grows. Both IP and the DBDD approaches scale much better as $L$ grows, with the latter finding the optimal solutions slightly faster. As the difficulty in the instances grows, the DBDD solution time grows at essentially the same pace as IP, which also elucidates the promise of investigated DBDDs in instances which lack structure. We remark that CPLEX is capable of solving quadratic models directly, which has a better performance than the methods presented here. Nonetheless, it transforms $Q$ into a positive semi-definite matrix, yielding a different model that we do not have access to.

\section{Conclusion}

This paper presents a novel decomposition-based optimization approach to binary optimization problems. The proposed technique combines the strength of binary decision diagrams, which are particularly successful at representing portions of problems with structure, with the strength of integer programming tech- 
nology. Computational experiments indicate promise in the approach for solving independent set and binary quadratic programming problems.

Acknowledgements: This research was supported in part by the Natural Sciences and Engineering Research Council of Canada (NSERC), Discovery Grant.

\section{References}

1. Akers, S.B.: Binary decision diagrams. IEEE Transactions on Computers C-27, 509-516 (1978)

2. Aldecoa, R., Marín, I.: Surprise maximization reveals the community structure of complex networks. Scientific Reports 3(1060) (2013)

3. Andersen, H.R., Hadzic, T., Hooker, J.N., Tiedemann, P.: A constraint store based on multivalued decision diagrams. In: Proceedings of the 13th international conference on Principles and practice of constraint programming. pp. 118-132. CP'07, Springer-Verlag, Berlin, Heidelberg (2007)

4. Balas, E.: Projection, lifting and extended formulation in integer and combinatorial optimization. Annals of Operations Research 140(1), 125-161 (2005), http://dx . doi.org/10.1007/s10479-005-3969-1

5. Behle, M.: Binary Decision Diagrams and Integer Programming. Ph.D. thesis, Max Planck Institute for Computer Science (2007)

6. Belik, I.: The analysis of split graphs in social networks based on the k-cardinality assignment problem. NHH Dept. of Business and Management Science Discussion Paper No. 2014/8, http://dx.doi.org/10.2139/ssrn.2405010 (February 2014)

7. Bergman, D.: New Techniques for Discrete Optimization. Ph.D. thesis, Tepper School of Business, Carnegie Mellon University (2013)

8. Bergman, D., Cire, A.A., Willem-Jan van Hoeve, J.: Discrete optimization with decision diagrams. To appear in INFORMS Journal on Computing (2015)

9. Bergman, D., Cire, A.A., Hoeve, W.J.v., Hooker, J.N.: Optimization bounds from binary decision diagrams. INFORMS Journal on Computing 26(2), 253-268 (2014)

10. Bergman, D., Cire, A., van Hoeve, W.J., Yunes, T.: Bdd-based heuristics for binary optimization. Journal of Heuristics 20(2), 211-234 (2014)

11. Bergman, D., van Hoeve, W.J., Hooker, J.: Manipulating MDD relaxations for combinatorial optimization. In: Achterberg, T., Beck, J. (eds.) Integration of AI and OR Techniques in Constraint Programming for Combinatorial Optimization Problems, Lecture Notes in Computer Science, vol. 6697, pp. 20-35. Springer Berlin / Heidelberg (2011)

12. Bliek, C., Bonami, P., Lodi, A.: Solving mixed-integer quadratic programming problems with ibm-cplex: a progress report. In: Proceedings of the Twenty-Sixth RAMP Symposium (2001)

13. Bramoull, Y., Kranton, R.: Public goods in networks. Journal of Economic Theory 135(1), $478-494$ (2007), http://www.sciencedirect.com/science/article/ pii/S0022053106001220

14. Bryant, R.E.: Graph-based algorithms for boolean function manipulation. IEEE Transactions on Computers C-35, 677-691 (1986)

15. Cire, A.A., van Hoeve, W.J.: Multivalued Decision Diagrams for Sequencing Problems. Operations Research 61(6), 1411-1428 (2013)

16. D. Bergman, A.A.C., van Hoeve, W.: Mdd propagation for sequence constraints. Journal of Artificial Intelligence Research 50, 697-722 (2014) 
17. Haus, U.U., Michini, C.: Representations of all solutions of boolean programming problems. In: ISAIM (2014)

18. Hu, A.J.: Techniques for efficient formal verification using binary decision diagrams. Thesis CS-TR-95-1561, Stanford University, Department of Computer Science (Dec 1995)

19. Johnson, M., Paulusma, D., van Leeuwen, E.: Algorithms to measure diversity and clustering in social networks through dot product graphs. In: Cai, L., Cheng, S.W., Lam, T.W. (eds.) Algorithms and Computation, Lecture Notes in Computer Science, vol. 8283, pp. 130-140. Springer Berlin Heidelberg (2013), http://dx.doi. org/10.1007/978-3-642-45030-3_13

20. Judd, S., Kearns, M., Vorobeychik, Y.: Behavioral conflict and fairness in social networks. In: Chen, N., Elkind, E., Koutsoupias, E. (eds.) Internet and Network Economics, Lecture Notes in Computer Science, vol. 7090, pp. 242-253. Springer Berlin Heidelberg (2011), http://dx.doi.org/10.1007/978-3-642-25510-6_21

21. Östergård, P.R.: A fast algorithm for the maximum clique problem. Discrete Applied Mathematics 120(13), 197 - 207 (2002), http://www.sciencedirect. com/science/article/pii/S0166218X01002906, special Issue devoted to the 6th Twente Workshop on Graphs and Combinatorial Optimization 